\title{
Mosaicism in Fanconi anemia: concise review and evaluation of published cases with focus on clinical course of blood count normalization
}

\author{
Eileen Nicoletti ${ }^{1}$ - Gayatri Rao ${ }^{1}$ Juan A. Bueren ${ }^{2,3,4}$ • Paula Río ${ }^{2,3,4}$ - Susana Navarro ${ }^{2,3,4}$ • Jordi Surrallés ${ }^{3,5,6}$. \\ Grace Choi ${ }^{1}$. Jonathan D. Schwartz ${ }^{1}$
}

Received: 25 July 2019 / Accepted: 6 February 2020 / Published online: 17 February 2020

(C) The Author(s) 2020

\begin{abstract}
Fanconi anemia (FA) is a DNA repair disorder resulting from mutations in genes encoding for FA DNA repair complex components and is characterized by variable congenital abnormalities, bone marrow failure (BMF), and high incidences of malignancies. FA mosaicism arises from reversion or other compensatory mutations in hematopoietic cells and may be associated with BMF reversal and decreased blood cell sensitivity to DNA-damaging agents (clastogens); this sensitivity is a phenotypic and diagnostic hallmark of FA. Uncertainty regarding the clinical significance of FA mosaicism persists; in some cases, patients have survived multiple decades without BMF or hematologic malignancy, and in others hematologic failure occurred despite the presence of clastogen-resistant cell populations. Assessment of mosaicism is further complicated because clinical evaluation is frequently based on clastogen resistance in lymphocytes, which may arise from reversion events both in lymphoid-specific lineages and in more pluripotent hematopoietic stem/progenitor cells (HSPCs). In this review, we describe diagnostic methods and outcomes in published mosaicism series, including the substantial intervals (1-6 years) over which blood counts normalized, and the relatively favorable clinical course in cases where clastogen resistance was demonstrated in bone marrow progenitors. We also analyzed published FA mosaic cases with emphasis on long-term clinical outcomes when blood count normalization was identified. Blood count normalization in FA mosaicism likely arises from reversion events in long-term primitive HSPCs and is associated with low incidences of BMF or hematologic malignancy. These observations have ramifications for current investigational therapeutic programs in FA intended to enable gene correction in long-term repopulating HSPCs.
\end{abstract}

Keywords Fanconi anemia $\cdot$ Bone marrow failure $\cdot$ Autologous stem cell transplantation $\cdot$ Gene therapy $\cdot$ Mosaicism

Electronic supplementary material The online version of this article (https://doi.org/10.1007/s00277-020-03954-2) contains supplementary material, which is available to authorized users.

Eileen Nicoletti

en@ rocketpharma.com

1 Rocket Pharmaceuticals, Inc., New York, NY, USA

2 Hematopoietic Innovative Therapies Division, Centro de Investigaciones Energéticas, Medioambientales y Tecnológicas (CIEMAT), Madrid, Spain

3 Centro de Investigación Biomédica en Red de Enfermedades Raras (CIBERER), Madrid, Spain
4 Instituto de Investigaciones Sanitarias Fundación Jiménez Díaz (IIS-FJD), Madrid, Spain

5 Genome Instability and DNA Repair Syndromes Group, Department of Genetics and Microbiology, Universitat Autònoma de Barcelona (UAB), Barcelona, Spain

6 Servicio de Genética e Instituto de Investigaciones Biomédicas del Hospital de Sant Pau, Barcelona, Spain 


\section{Fanconi anemia and mosaicism}

\section{Fanconi anemia overview}

Fanconi anemia (FA) is a deoxyribonucleic acid (DNA) repair disorder resulting from mutations in genes encoding for protein components of the FA/BRCA DNA repair pathway. More than 20 identified proteins interact as components of the FA core, FANCI, FANCD2 and downstream complexes. In conjunction with other DNA repair elements, FA proteins facilitate repair of interstrand crosslinks (ICLs) and other forms of DNA damage $[1,2]$. FA proteins also contribute to other cellular processes beyond DNA repair, including cellular responses to oxidative stress and inflammatory stimuli [3-5].

FA is characterized by frequent bone marrow failure (BMF), myeloid malignancies, and epithelial cancers $[1,6]$. The BMF and neoplastic manifestations of FA are frequently but not universally accompanied by congenital abnormalities including growth retardation/short stature, microcephaly, and abnormalities of skin pigmentation, upper limbs (especially thumbs and radii), gonads, kidneys, and other organ systems. Manifestations of BMF are frequently noted during the first decade of life; the incidences of myeloid and solid organ malignancies increase during the second and third decades of life, respectively, such that the cumulative incidence of acute myeloid leukemia (AML)/myelodysplastic syndrome (MDS) is approximately $30 \%$ by age 30 and the cumulative incidence of solid organ cancers approaches $40 \%$ by age $40[6,7]$.

\section{Fanconi anemia mosaicism}

Somatic mosaicism in FA (hereafter referred to as mosaicism) arises from reversion or other compensatory mutations in hematopoietic stem cell/progenitor cells (HSPCs) from which arise a population of bone marrow and blood cells with a functional DNA repair capacity. Patients in whom a reversion in a pathogenic FA mutation has occurred frequently have two distinct blood cell populations. One population is sensitive to DNA-damaging agents (mitomycin-C [MMC] or diepoxybutane [DEB]) and consistent with an FA diagnosis and another population resistant to these DNA-damaging (or clastogenic) agents - hence the "mosaic" label indicating the coexistence of phenotypically distinct cellular components [8-10]. The purpose of this review is to summarize the existing literature regarding FA mosaicism, with particular emphasis on persistent diagnostic and prognostic uncertainties. We provide an evaluation of case series in which FA mosaic patients were followed clinically over years (and at times decades), with emphasis on the clinicopathologic relevance of laboratory diagnostic evaluations of these cases. Additionally, we conducted a literature-based assessment of FA mosaic cases most likely emanating from reversion or other compensatory mutations in long-term repopulating HSC populations.

Mosaicism in FA patients was noted prior to initial identification of the first FANC gene (FANCC) in 1992 [11], with initial reports describing FA patients in whom $60-80 \%$ of cultured lymphocytes (either PHA-stimulated T cells or EBV-transformed B cells) displayed resistance to concentrations of alkylating agents typically toxic to FA patient cells $[12,13]$. Several mechanisms by which an additional genetic event may result in a functional FA gene and protein have been identified and include gene conversion, intragenic crossover, back mutation, and second-site mutation - each of which may result in a restoration of the affected gene to wildtype $[8,14,15]$. Second-site mutations involve either a compensatory point mutation (insertion or deletion) at a distinct site within a mutated gene, resulting in a functional gene and protein that may be nonetheless distinct from wildtype [15-17]. The mechanisms by which these various mutations may result in cell populations expressing a functional gene and/or protein are illustrated in Fig. 1.

\section{Mosaicism in other genetic disorders}

Reversion mutations and resultant mosaicism have been reported in Bloom syndrome, X-linked and adenosine deaminase severe combined immunodeficiency (X-SCID and ADA-SCID), Diamond Blackfan anemia (DBA), WiskottAldrich syndrome, epidermolysis bullosa, tyrosinemia, and dyskeratosis congenita (DC) [18-25]. The extent of phenotypic correction in any clinical setting is dependent on both the chronology of a reversion event either during embryonic or postnatal development and the degree by which a disorder manifests in hierarchical (as opposed to heterarchical) organ systems. Disorders predominantly affecting blood cells may be particularly reversible because of the hierarchical nature of hematopoiesis, with partial or comprehensive phenotypic corrections identified most frequently in the context of normalization within a single lineage [19-21]. Multilineage mosaicism has been reported in DC [25]. In several of these conditions and in FA in particular, the underlying disorder predisposes to heightened genetic instability, enhancing the potential for additional mutations, many of which are potentially deleterious (resulting in characteristic frequent malignancy in Bloom syndrome, DBA, DC, and FA), but in more limited instances may confer a beneficial phenotypic reversion. A particularly unstable intragenic duplication was recently characterized in a FANCB patient with mosaicism associated with mild hematologic deficits. The instability of this duplication was evident not only from a decreasing percentage of aberrant blood cells over 11 years of observation but from the presence of a modest population $(8 \%)$ of fibroblasts displaying a wild-type allele, 


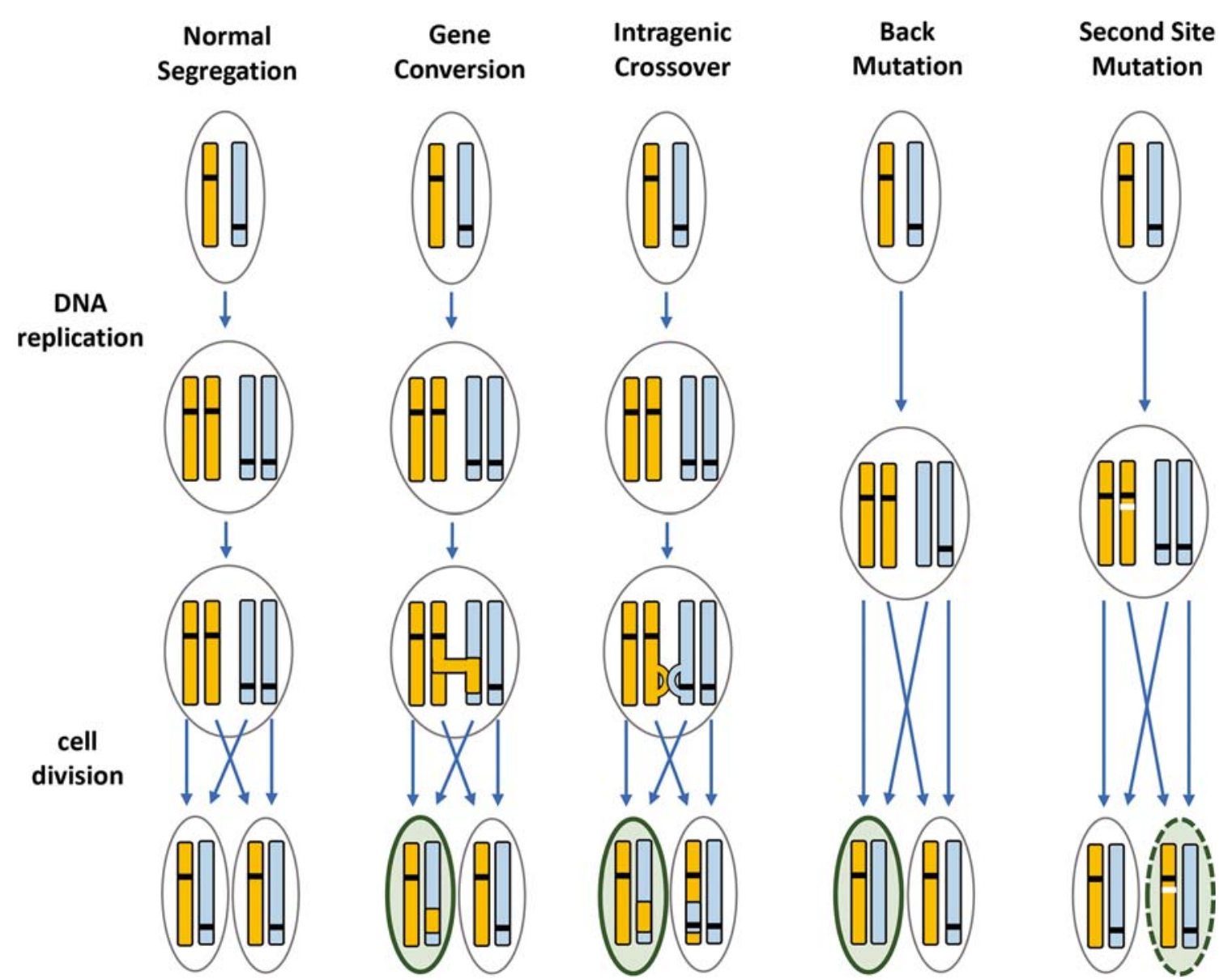

Fig. 1 Several mechanisms by which additional chromosomal rearrangements or mutations may facilitate correction of an inherited recessive genetic disorder. The yellow and blue bars indicate an individual gene, with black hashes indicating a disease-causing mutation. Reversion mutations may arise either during or subsequent to DNA replication and may involve transfer of genetic material between paired chromosomes (gene conversion or intragenic crossover) or

mutations within a single chromosome (and gene). Gene conversion, intragenic crossover, and back mutations result in genetic correction in one allele within a daughter cell (indicated by the green border and background). Second-site mutations (white hash) result in a gene capable of generating a functional protein (indicated by the green dashed border and green background) in one allele, although the gene itself may differ from wildtype. Adapted from Pasmooij et al. [17]

representing the first identification of reversion in a nonhematopoietic cell population in FA [26].

\section{FA mosaicism: selective growth advantage and prognostic uncertainty}

In the abovementioned diseases, mosaicism has been described as "natural gene therapy" [27, 28], and a selective growth advantage of revertant cells in selected disorders and organ systems (including the hematologic component of FA) has long been hypothesized. The potential for a marked proliferative advantage at the HSC level has been most profoundly illustrated by a case of monozygotic twin sisters diagnosed with FA early in life who nonetheless had demonstrated hematologic stability during 28 years of reported follow-up, with clastogen resistance evident in peripheral blood lymphocytes despite characteristic fragility of cultured fibroblasts. Analysis of bone marrow progenitors indicated multilineage presence of the compensatory mutation, consistent with a hypothesized prenatal reversion in a long-term HSC, resulting in multilineage engraftment via shared in utero circulation $[28,29]$. This example of gene reversion and selective growth advantage enabling comprehensive and sustained hematopoiesis has represented a naturally occurring proof of concept for the potential hematologic correction of FA via gene therapy. A selective advantage of gene-corrected FA cells has been demonstrated in murine FA models [30-32], human embryonic stem cells [33], induced pluripotent stem cells [34], and gene-corrected cells from FA patients transplanted in immunocompromised mice [35]; these observed in vivo selective advantages have been largely facilitated by administration of cytotoxic conditioning agents. A selective advantage has also recently been demonstrated in nonconditioned FA-A patients receiving gene-corrected 
autologous CD34+ cells, by means of progressive increases in gene-corrected and clastogen-resistant lymphocytes and marrow progenitors during 18-30 months of follow-up [36].

The presence of a revertant FA hematopoietic cell population has often been associated with a favorable hematologic course in FA patients. However, the presence of clastogenresistant blood and marrow cells may not uniformly be associated with long-term BMF- or AML-free survival. Gregory and colleagues reported a male patient with hematologic stability during the initial 9-17 years of life and a stable population of DEB-resistant peripheral blood lymphocytes (61$74 \%$ ). Analysis of bone marrow progenitors at age 15 indicated multilineage presence of the revertant mutation (including lymphoid, myeloid, and erythroid progenitors). At ages 15 and 17, this patient nonetheless developed evidence of an $11 \mathrm{q}$ deletion in a population of non-revertant bone marrow cells - a cytogenetic abnormality potentially associated with myeloid malignancy [37]. The prognostic uncertainty regarding the presence of a hematopoietic population containing a reversion mutation is rendered additionally complex because the diagnostic methods utilized in FA are most frequently directed at a differentiated T-lymphoid cell population that may be genetically divergent from multilineage stem and progenitor populations. The diagnosis and clinical prognosis of FA mosaic patients during recent decades are detailed subsequently.

\section{Mosaicism diagnosis}

Diagnosis of mosaicism in FA is contingent on demonstration of a population of blood and/or bone marrow cells that display resistance to concentrations of DNA-damaging agents that are typically toxic to FA hematopoietic cells. Diagnostic challenges persist because mosaicism may be restricted to specific cell lineages and evaluation of multiple blood and marrow lineages is frequently not clinically feasible. The percentage of resistant cells within a single lineage may not be indicative of the overall hematologic milieu and (especially at a single timepoint) may not provide clinically meaningful prognostic information.

A critical component of an FA diagnosis and of potential FA mosaicism involves evaluation of cultured $\mathrm{T}$ lymphocytes in the presence of DNA-damaging agents, most frequently DEB or MMC. Lymphocytes from FA patients frequently display marked chromosomal instability (also referred to as fragility, evidenced by chromosomal breaks and radial figures) in the presence of clastogen concentrations that result in minimal abnormalities in non-FA cells $[9,10]$. Some centers, including those involved in the evaluation of a large and rigorously followed Spanish FA mosaic population, have proposed diagnostic algorithms classifying individuals with fewer than $20 \%$ aberrant cells cultured in $0.1-\mu \mathrm{g} / \mathrm{ml}$ DEB as nonFA patients, $20-40 \%$ as consistent with FA mosaics, $>60 \%$ as consistent with FA, and $40-60 \%$ as possible mosaicism, with additional information regarding the number of breaks per cell further contributing to diagnosis [10]. Specific DNAdamaging agents, concentrations, other culture and cell selection conditions, and threshold aberrancy levels have varied among investigative centers such that no single standard for a determination of mosaicism exists. The presence of divergent DEB resistance between a patient's lymphocytes and cultured fibroblasts is considered consistent with FA mosaicism, as is an increasing percentage of DEB-resistant lymphocytes over sequential intervals. A German-led international consortium of investigative centers has also identified mosaicism by decreased clastogen stimulation of G2-phase arrest [15]; French investigators have described normalized FANCD2 ubiquitination in the presence of clastogens as indicative of mosaicism [38]. As will be discussed subsequently, results of DEB-chromosomal stability evaluations in peripheral blood lymphocytes may not necessarily be indicative of reversions in other hematopoietic compartments, including multipotent HSPCs [14].

Gene sequencing has become an increasingly valuable and utilized modality for the confirmation of an FA diagnosis; however, sequencing of distinct blood and marrow progenitor cell populations has been performed infrequently and continues to be a predominantly research-focused modality [28, 37]. Evaluation of the MMC resistance of cultured bone marrow-derived colony forming cells (CFCs) has emerged as a complementary test for identifying phenotypic reversion in progenitor populations. The number of investigations in which this parameter has been correlated with long-term clinical outcomes is extremely limited [28, 37, 39]; however, the increasing percentage of MMC-resistant bone marrow $\mathrm{CFCs}$ has been identified in recent autologous gene therapy clinical trials and has been recognized as a relevant indicator of genetic and phenotypic reversal in pluripotent hematopoietic progenitor cells [36]. Ongoing discussions involving international multidisciplinary investigators have increasingly identified MMC resistance of bone marrow progenitors as meaningful and potentially objective clinical phenomena in either FA mosaicism or autologous hematopoietic gene therapy settings [40, 41]. Ongoing consensus discussions have also indicated the potential importance of evaluations employing multiple MMC concentrations, serial evaluations of MMC resistance over time, and corroboration of clastogen resistance with functional assays such as FANCD2 ubiquitination. When feasible, genetic sequencing and multilineage assessment are additionally relevant for comprehensive mosaicism assessment.

\section{Clinical outcomes in FA mosaic patients}

In addition to the two illustrative and deeply investigated FA mosaic cases described previously, several additional series published over a 16-year period (1997-2013) have detailed the clinical course and essential laboratory correlates in FA 


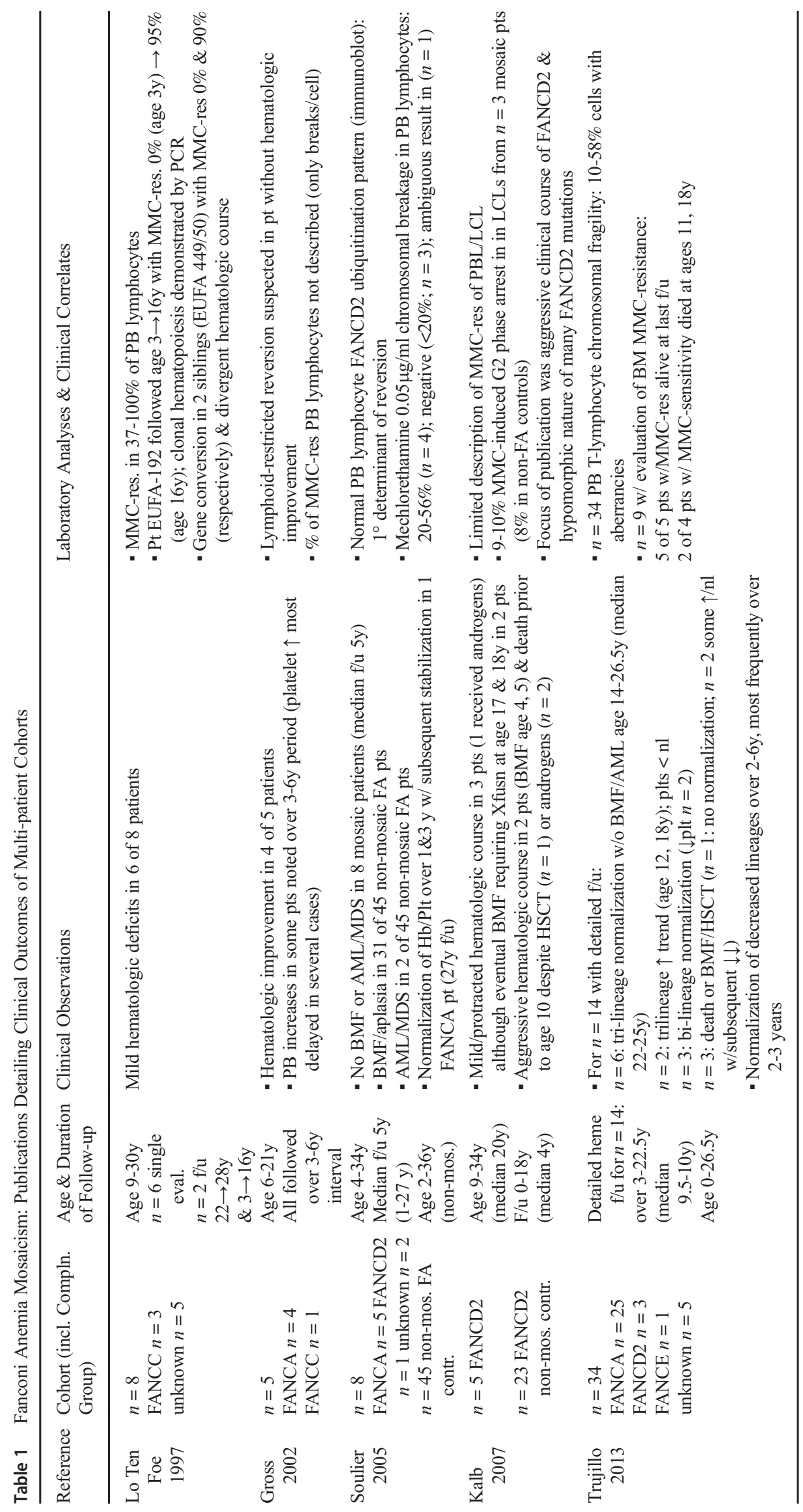


mosaic patients. These include 4 publications and a published doctoral thesis, describing outcomes in FA mosaic patients (range: $n=5-14$ per publication) [8, 14, 15, 38, 39]. Detailed information regarding these series is provided in Table 1. Essential themes emerging from these cohorts include the following:

1. FA mosaicism has largely been reported in cases arising from more frequently observed mutations in FA core and ubiquitination complex genes (predominantly FANCA, $F A N C C$, and FANCD2); there is limited published information on FA mosaicism arising from mutations in downstream or more recently discovered FA genes/proteins [26, 42, 43].

2. Hematologic stability and improvement have been noted in a single lineage, or frequently in bi- and trilineage settings, consistent with the hypothesis that reversion mutations arise across a spectrum of pluripotent or more committed HSPCs [39].

3. A broad range of peripheral blood lymphocyte resistance to DEB (or other clastogens) has been reported, with a limited number of studies showing correlation between the percentages of resistant PB cells and clinical outcomes. (Of note, T lymphocyte mosaicism has been reported in up to $15-20 \%$ of FA patients and had been frequently associated with HSCT engraftment failure prior to the incorporation of fludarabine-based conditioning) [44].

4. FA mosaicism has been frequently but not universally associated with stable or increasing hematologic parameters over prolonged follow-up.

5. In the limited settings where normalization of blood counts has been observed over time, increases in blood lineages have been reported over a 1- to 6-year interval (most frequently over 2-3 years), and multilineage increases have been observed in non-contemporaneous (i.e., staggered) sequences as indicated in Table 1 [14, 38, 39].

6. In the limited settings where normalization of blood counts has been observed over time, platelet normalization has at times been the most indolent and incomplete relative to other lineages, with some increases resulting in levels below normal limits but not requiring clinical intervention, as indicated in Table 1 [14, 39].

7. Evaluation of MMC resistance in bone marrow CFCs has been undertaken infrequently. In the one series in which BM MMC resistance was evaluated, 5 of 5 patients with documented MMC resistance were alive and without hematologic complications at last follow-up (with stable clinical course over 9-15 years in 3 patients for whom this information was available) and 2 of 4 patients in whom marrow CFCs were sensitive to MMC died at ages 11 and 18 of BMF and unspecified complications [39].
An additional investigation based on these and other published FA mosaic cases is described in a subsequent section of this review. We undertook this literature-based investigation with an intent of identifying a subset of FA mosaic patients most likely to harbor reversion mutations in long-term repopulating $\mathrm{HSCs}$.

\section{Hypomorphic mutations in FA}

Relatively little has been reported regarding the incidence or clinical significance of hypomorphic mutations in FA, in which the impacted protein is expressed with reduced quantity and/or function. Hypomorphic mutations have been described in FANCD2 and were not associated with any reduction in the typically aggressive hematologic manifestations associated with this complementation group; the incidence of mosaicism in FANCD2 also appears comparable to that in FANCA or other core complex FA groups [15].

Recently, a cohort of 11 Sicilian patients with FANCA hypomorphic mutations were reported, in which mutations resulted in FANCA proteins that localized to cytoplasm and facilitated more normalized mitochondrial function (relative to cells lacking FANCA), with peripheral blood-derived lymphoblast sensitivity comparable to non-mosaic FA patients. The clinical course of this cohort ranged from indolent ( $n=$ $3)$ to severe $(n=2)$, with the majority of patients $(n=6)$ encountering moderate single-, bi-, or trilineage cytopenias and receiving allogeneic hematopoietic transplant at ages 9-21 [45]. It is likely that as more comprehensive genetic sequencing becomes clinically feasible, additional hypomorphic mutations may be recognized, including some with potential for more indolent clinical progression resemblant of mosaicism, but without the clastogen resistance associated with compensatory mutations in FA. Additional investigations into the mitochondrial and other "non-canonical" functions of FA proteins may also elucidate potential prognoses associated with these uncommon variants [46].

\section{Review of FA mosaic publications with emphasis on blood count normalization}

\section{Overview and objectives}

The clinical significance of FA mosaicism remains uncertain more than 20 years after this phenomenon was initially reported. We sought a comprehensive literature-based understanding of FA mosaicism with emphasis on cases most likely emanating from reversion mutations in long-term repopulating HSC populations. Focusing on the minority of FA mosaic cases in which normalization of all peripheral blood lineages was reported, we identified a population of FA mosaic patients 
in whom there appears to be high likelihood of multi-decade BMF-free and leukemia-free survival.

Our objectives were to identify FA mosaicism cases reported in the medical literature for which there was information regarding individual patient clinical outcomes and to further identify the subset of FA mosaic cases in which there was normalization of all peripheral blood lineages (because such cases likely result from reversion mutations in long-term repopulating HSC populations) and to determine clinical course in this clinical subpopulation. In particular, we sought to quantify the incidences of BMF, hematologic malignancy, and other adverse outcomes in FA mosaic patients, including those with blood count normalization and those with no or incomplete normalization.

\section{Methods}

We identified published FA mosaic cases via PubMed searches, reference citations, and a publicly available doctoral thesis on this specific condition. A database was constructed from cases in which there was sufficient clinical information enabling evaluation of age at FA and mosaic diagnosis, duration of follow-up and development of BMF, malignancy, allogeneic hematopoietic stem cell transplant (HSCT), survival, and cause of death. Blood count normalization was defined as improvement in absolute neutrophil count (ANC) to at least $1000 / \mathrm{mm}^{3}$, hemoglobin to at least $9 \mathrm{~g} / \mathrm{dL}$, and platelets to at least $50,000 / \mathrm{mm}^{3}$ after previously lower levels or maintenance of these levels over multiple intervals in settings where earlier counts were not available or presence of these levels at a single timepoint with prior/subsequent hematologic stability reported by the investigator or blood counts normalized or within normal range per investigator without precise values specified.

\section{Results}

Figure 2 details the literature search results in which 51 publications describing FA mosaicism were identified between 1976 and 2018, of which 23 publications ( $n=123$ patients) contained clinical information. A subset of 37 patients (30\%) had normalization of blood counts (including 19 for whom detailed blood count information over multiple timepoints was reported). Fifteen FA mosaic patients were reported in whom no or incomplete normalization (including normalization of 1 or 2 lineages) was described. Blood count information was not reported in 71 patients. The subgroup of 37 FA mosaic patients with normalized blood counts were reported from investigators in Spain $(n=13)$, Germany $(n=10)$, France $(n=8)$, the USA $(n=5)$, and Japan $(n=1)$. Complementation groups of patients in this cohort were Group A $(n=24)$, Group B $(n=1)$, Group C $(n=1)$, Group $\mathrm{E}(n=1)$, Group T $(n=1)$, and not reported/known $(n=9)$. Publications detailing these patients are shown in Supplemental Table 1. As indicated previously, there is limited published information on FA mosaicism arising from

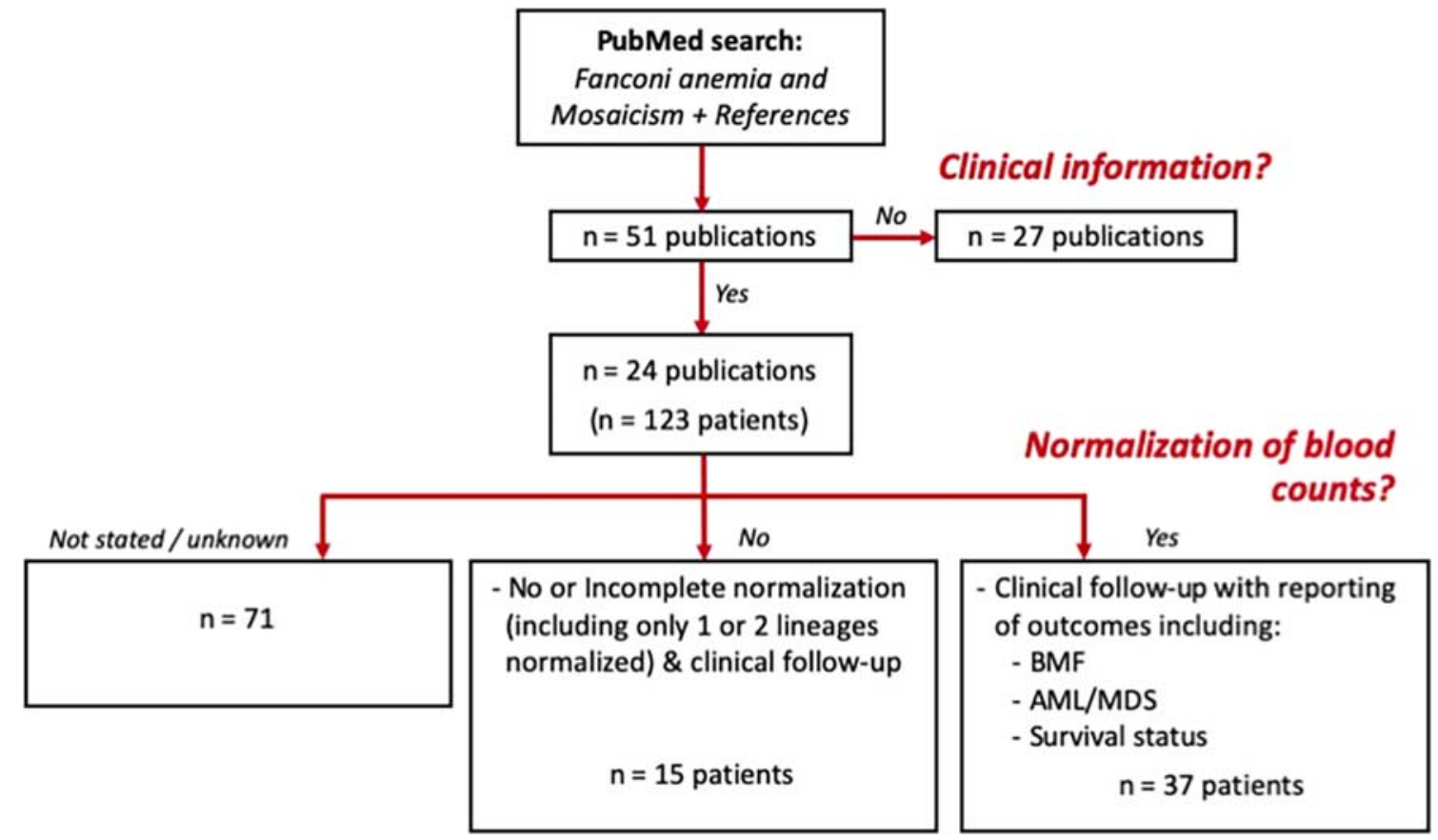

Fig. 2 In the cohort of FA mosaic patients $(n=37)$ with normalized blood counts and information regarding clinical outcomes, individuals were included from the following cohorts: Spain $(n=13)$, Germany $(n=10)$, France $(n=8)$, the USA $(n=5)$, and Japan $(n=1)$. Complementation groups of patients identified in this cohort included: Group A $(n=24)$, Group B $(n=1)$, Group C $(n=1)$, Group E $(n=1)$, and Group T $(n=1)$; in $n=7$ patients, complementation group was unknown 


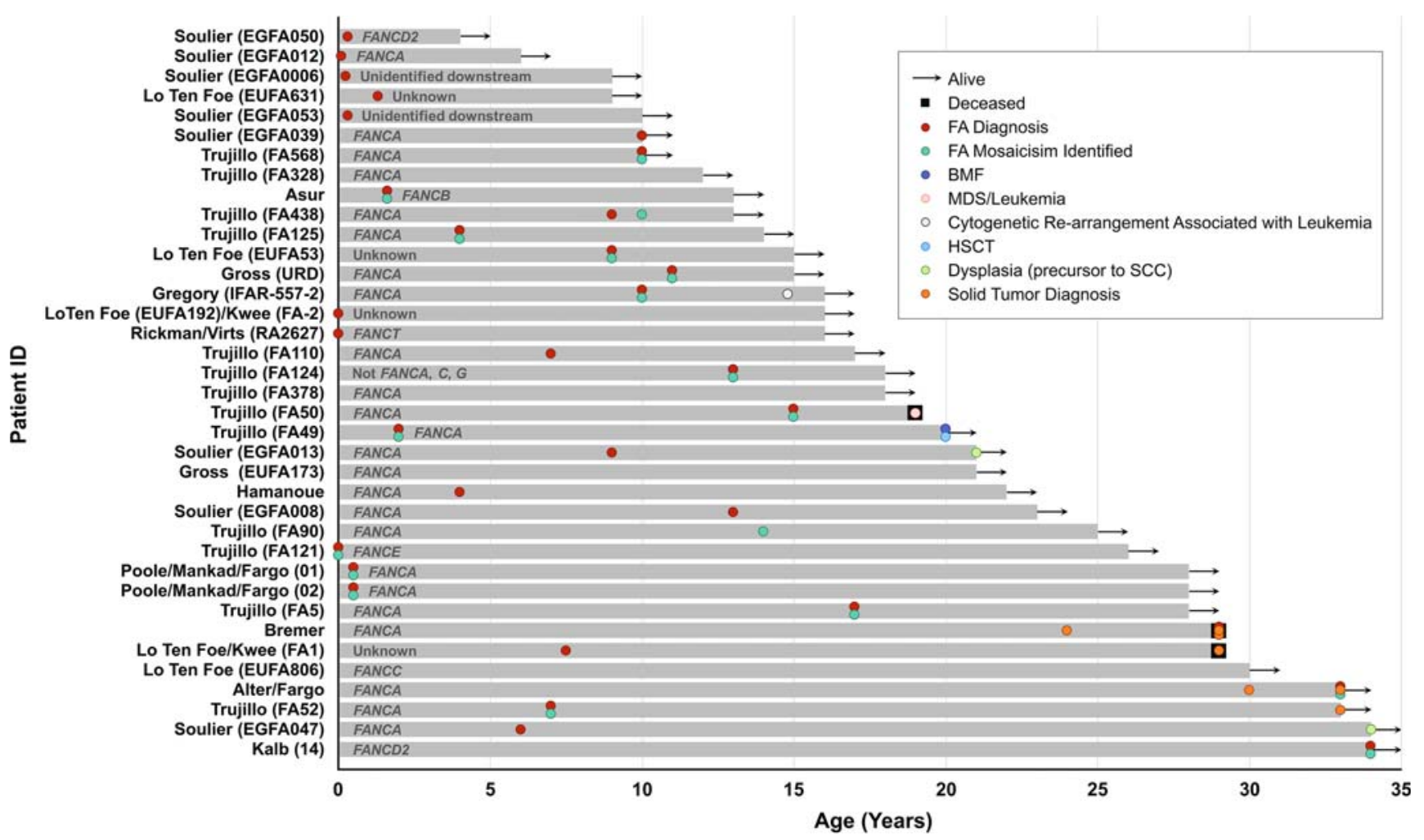

Fig. 3 Swimmer plot depicting clinical course for 37 FA mosaic patients with normalized blood counts, including age at diagnosis (when available), last follow-up, and occurrences of BMF, AML/MDS, allogeneic HSCT, and solid organ cancers. The majority of patients (34

mutations in downstream or more recently discovered FA genes/proteins.

The clinical outcomes for the 37 FA mosaic patients with normalized blood counts are depicted in Fig. 3. Median follow-up within this subgroup was to age 18 (range 434 years). Thirty-four patients $(92 \%)$ were alive at last follow-up. Death in 3 patients occurred at ages 19 (AML), 29 of 37) were alive without BMF, malignancy, or transplant at last followup. Criteria for normalization are provided in the text and are in general more stringent (exclusive) than those applied by investigators in Table 1

(lung cancer), and 29 (head and neck cancer). BMF was reported in one patient (3\%, age 20) and leukemia (AML/MDS) in one patient (3\%, age 19). An additional patient developed an $11 \mathrm{q}$ deletion (MLL rearrangement) in a clonal population persistent over 2 years of follow-up but without overt evidence of leukemia. Solid organ malignancies were reported in 4 patients (11\%, ages 24-33) including 2 patients with
Table 2 Clinical outcomes for Fanconi anemia mosaic patients

\begin{tabular}{llll} 
A. Mosaic with blood count normalization $(n=37)$ & \\
\hline Status & $n(\%)$ & Age (y) & Median: 18 Range: 4-34 \\
\hline Alive at last follow-up & $34(92 \%)$ & At last follow-up: & $19,29,29$ \\
Deceased at follow-up & $3(9 \%)$ & At death: & 20 \\
BMF & $1(3 \%)$ & At BMF diagnosis: & 19 \\
AML/MDS & $1(3 \%)$ & At AML/MDS diagnosis: & Median: 29 Range: 24-33 \\
Solid organ malignancy & $4(11 \%)$ & At cancer diagnosis: & \\
& & & \\
B. Mosaic with no/incomplete blood count normalization (n=15) & Median:14 Range: 8-47.5 \\
Status & $n(\%)$ & Age (y) & Range: 9-39 \\
Alive at last follow-up & $8(53 \%)$ & At last follow-up: & Median: 9.5 Range: 0-47.5 \\
Deceased at follow-up & $7(47 \%)$ & At death: & $17.5,47.5$ \\
BMF & $12(80 \%)$ & At BMF diagnosis: & 38,39 \\
AML/MDS & $2(13 \%)$ & At AML/MDS diagnosis: \\
Solid organ malignancy & $2(13 \%)$ & At cancer diagnosis: &
\end{tabular}


multiple sequential cancer diagnoses. Additional details regarding these outcomes are provided in Table 2. The clinical outcomes for the 15 FA mosaic patients in whom no or incomplete normalization was reported were more adverse and are provided in Table $2 ; 8$ of these patients (53\%) were alive at last follow-up with 7 deaths occurring between ages 9 and 39 . BMF was reported in 12 of 15 patients $(80 \%$, median age 9.5 years); 2 of 15 patients (13\%) developed AML/MDS, and $2(13 \%)$ developed solid organ malignancies.

FA mosaic patients with blood count normalization were less likely to develop BMF ( $p<0.0001$, Fisher's Exact Test, 2sided) and more likely to remain alive $(p=0.0034)$ relative to FA mosaics without normalization. A lower percentage of mosaic patients with normalization developed hematologic malignancy ( $3 \%$ vs. $14 \%$ ), but this difference was not statistically significant. The incidences of solid organ malignancies were similar between these subgroups.

Based on these findings, FA mosaicism with normalized blood counts appears to occur in a minority (30\%) of patients whose peripheral lymphocyte assessments indicate resistance to DNA-damaging agents via standard testing; the potential for reporting bias is such that the actual incidence may be lower; it is likely that fewer than $5 \%$ of all FA patients experience blood count normalization as a result of reversion/ compensatory mutations. FA mosaicism with normalized blood counts is associated with very limited incidences of BMF or hematologic malignancy, limited requirement for allogeneic HSCT, and relatively limited mortality during the initial 2-4 decades of life. Solid tumors arise during the $3 \mathrm{rd}$ and 4th decades of life in FA mosaic patients regardless of blood count normalization, as is the case with non-mosaic FA patients. Two of four FA patients with normalized blood counts who developed solid tumors had a second malignancy diagnosed within several years of the initial cancer diagnosis; solid tumors were fatal in at least 2 of 4 reported patients.

\section{Discussions and summary}

These findings indicate that there is a population of FA mosaic patients with very limited risk of BMF or hematologic malignancy, likely as a result of reversion mutations in primitive HSPC populations. Limitations for conclusive analysis result from the absence of bone marrow-based genetic or mitomycin-resistance evaluation in the overwhelming majority of FA mosaic cases. Analysis is also limited because sequential chronologic assessment of peripheral blood or bone marrow clastogen resistance has been performed infrequently. It is very likely that FA mosaicism with normalized blood counts is the result of mutations in long-term HSPCs, but it is not known what percentage of HSPC with reversion events is required to enable stable or normalized peripheral blood cell counts [28, 37]. Presumably those mosaic patients in whom no or incomplete normalization occurred were diagnosed as a result of reversions in committed progenitor (lymphoidinclusive) lineage or short-term repopulating progenitor cells, but more detailed genetic evaluation of progenitor lineages will be needed to validate this hypothesis. Additional nongenetic elements (including epigenetic factors, stromal microenvironment, telomeric depletion, and aldehyde exposure and metabolism) may also contribute to hematopoietic stability in settings of genetic correction [5, 47, 48]. It is plausible that reversion events may only result in favorable clinical outcomes when these take place in long-term repopulating HSCs and additional favorable metabolic or other nongenetic conditions. A case of mosaicism emerging subsequent to initiation of androgen therapy has been reported, with blood count normalization persisting subsequent to androgen discontinuation (duration undetermined); it is plausible that androgens may selectively enhance hematopoiesis in dormant revertant HSCs and that some cases of prolonged androgenattributed hematologic benefit were in fact the result of undetected mosaicism [49]. Prospective translational studies will be required to enable optimal understanding of genotype/ phenotype correlations in settings of reversion mutations or therapeutic genetic correction - these are planned in the context of the next generation of gene therapy clinical investigations.

Clinical trials of autologous gene therapy in FA have been conducted during the prior decades, with most recent results indicating genetic correction and reversal of sensitivity to DNA-damaging agents at levels resembling those seen in FA mosaic patients $[35,50,51]$. Our findings also provide rationale for further clinical evaluation of gene therapy because they indicate that there is a likely threshold of genetic and phenotypic correction above which multi-decade BMF-free survival may be likely. These comparisons between FA mosaic and gene therapy treated patients are limited in that mosaicism likely results from a single-cell mutation (or mutation within a very finite subset of cells), whereas gene therapy involves introduction of a likely higher number of genecorrected HSPCs with oligoclonal hematopoietic repopulation observed to date [36]. The long-term significance of the oligoclonal hematopoiesis identified in nonconditioned gene therapy recipients (and suspected in FA mosaic patients) is uncertain, although there exist similarities to the clonal hematopoiesis that has become increasingly recognized as a distinct phenomenon, particularly in elderly individuals. Clonal hematopoiesis in the elderly occurs frequently and, even in the absence of mutations identified with hematologic malignancy, is associated with increased risk of hematologic malignancy and cardiovascular events, although the absolute risk in individual patients is relatively limited $[52,53]$. Long-term follow-up studies of the initial gene therapy treated FA patients are underway, although multi-year observation will likely be required to enable definitive conclusions regarding the ramifications of oligoclonal hematopoiesis. 
In summary, FA mosaicism is a condition in which varied and frequently divergent degrees of resistance to DNA damage are present within the hematopoietic system of an individual patient, or (more rarely) the entire hematopoietic system displays evidence of normal or near-normal resistance to DNA damage despite clastogen sensitivity in non-hematopoietic tissues. These findings are at times accompanied by normalized or stable peripheral blood counts, with some patients surviving into adulthood without bone marrow failure or hematologic malignancy. Some degree of mosaicism is likely present in 15-20\% of FA patients, although the incidence of reversion mutations in primitive HSCs resulting in sustained hematologic stability likely occurs in fewer than $5 \%[9,10,15,38]$. Our additional evaluation of 37 cases with documented blood cell count normalization suggests that there is a population of FA patients in whom a sufficient genetic reversion and selective advantage enable hematologic stability through at least three decades with limited BMF or myeloid malignancy. The longterm results of gene therapy studies - especially those involving no conditioning - are likely to provide important information as to the clinical relevance of reversion mutations and other forms of genetic correction in this complex disorder.

Authors' contribution EN performed the literature review and collected the data. GR performed the literature review and collected the data. JAB contributed to the interpretation of data and provided critical review of the manuscript. PR contributed to the interpretation of data and provided critical review of the manuscript. SN contributed to the interpretation of data and provided critical review of the manuscript. JS contributed to the interpretation of the data and provided critical review of the manuscript. GC performed analysis of data and designed the figures. JDS conceived the project, performed the analysis, and wrote the manuscript. All authors have read and approved the final manuscript.

\section{Compliance with ethical standards}

Conflict of interest Eileen Nicoletti is an employee of Rocket Pharmaceuticals, Inc. Gayatri Rao is an employee of Rocket Pharmaceuticals, Inc. Juan A. Bueren is the head of the Hematopoietic Innovative Therapies Division at Centro de Investigaciones Energeticas, Medioambientales y Tecnologicas (CIEMAT) which receives funding for Fanconi Anemia (FA) gene therapy and has licensed the PGK-FANCAWpre* lentiviral vector to Rocket Pharmaceuticals, Inc. He is also a consultant for Rocket Pharmaceuticals, Inc. Paula Río is a member of the Hematopoietic Innovative Therapies Division at Centro de Investigaciones Energeticas, Medioambientales y Tecnologicas (CIEMAT) which receives funding for Fanconi Anemia (FA) gene therapy and has licensed the PGK-FANCA-Wpre* lentiviral vector to Rocket Pharmaceuticals, Inc. Susana Navarro is a member of the Hematopoietic Innovative Therapies Division at Centro de Investigaciones Energeticas, Medioambientales y Tecnologicas (CIEMAT) which receives funding for Fanconi Anemia (FA) gene therapy and has licensed the PGK-FANCAWpre* lentiviral vector to Rocket Pharmaceuticals, Inc. Jordi Surrallés does not have any conflicts of interest. Grace Choi is an employee of Rocket Pharmaceuticals, Inc. Jonathan D. Schwartz is an employee of Rocket Pharmaceuticals, Inc.

Ethical approval This article does not contain any studies with human participants or animals performed by any of the authors.
Open Access This article is licensed under a Creative Commons Attribution 4.0 International License, which permits use, sharing, adaptation, distribution and reproduction in any medium or format, as long as you give appropriate credit to the original author(s) and the source, provide a link to the Creative Commons licence, and indicate if changes were made. The images or other third party material in this article are included in the article's Creative Commons licence, unless indicated otherwise in a credit line to the material. If material is not included in the article's Creative Commons licence and your intended use is not permitted by statutory regulation or exceeds the permitted use, you will need to obtain permission directly from the copyright holder. To view a copy of this licence, visit http://creativecommons.org/licenses/by/4.0/.

\section{References}

1. Shimamura A, Alter BP (2010) Pathophysiology and management of inherited bone marrow failure syndromes. Blood Rev 24(3):101122 Erratum in: blood rev 2010; 24(4-5):201

2. Kee Y, D'Andrea AD (2012) Molecular pathogenesis and clinical management of Fanconi anemia. J Clin Investig 122:3799-3806

3. Du W, Rani R, Sipple J, Schick J, Myers KC, Mehta P et al (2012) The FA pathway counteracts oxidative stress through selective protection of antioxidant defense gene promotors. Blood 119(18): $4142-4151$

4. Zhang H, Kozono DE, O'Connor KE, Vidal-Cardenas S, Rousseau A, Hamilton A et al (2016) TGF- $\beta$ inhibition rescues hematopoietic stem cell defects and bone marrow failure in Fanconi anemia. Cell Stem Cell 18(5):668-681

5. Bagby G (2018) Recent advances in understanding hematopoiesis in Fanconi anemia. F1000 Res 7(F1000 faculty rev):105. 17 Jul 2019 update. https://doi.org/10.12688/f1000research.13213.1

6. Kutler DI, Singh B, Satagopan J, Batish SD, Berwick M, Giampietro PF, Hanenberg H, Auerbach AD (2003) A 20-year perspective on the international Fanconi Anemia registry (IFAR). Blood 101:1249-1256

7. Alter BP, Giri N, Savage SA, Rosenberg PS (2018) Cancer in the National Cancer Institute inherited bone marrow failure syndrome cohort after fifteen years of follow-up. Haematologica 103(1):30 39

8. Lo Ten Foe JR, Kwee ML, Rooimans MA, Oostra AB, Veerman AJ, van Weel M et al (1997) Somatic mosaicism in Fanconi anemia: molecular basis and clinical significance. Eur J Hum Genet 5(3): 137-148

9. Fargo JH, Rochowski A, Giri N, Savage SA, Olson SB, Alter BP (2014) Comparison of chromosome breakage in non-mosaic and mosaic patients with Fanconi anemia, relatives, and patients with other inherited bone marrow failure syndromes. Cytogenet Genome Res 144(1):15-27

10. Castella M, Pujol R, Callén E, Ramirez MJ, Casado JA, Talavera M et al (2011) Chromosome fragility in patients with Fanconi anemia: diagnostic implications and clinical impact. J Med Genet 48:242250

11. Strathdee CA, Gavish H, Shannon WR, Buchwald M (1992) Cloning of cDNAs for Fanconi's anaemia by functional complementation. Nature 356(6372):763-767 Erratum in Nature 1992; 358(6385):434

12. Auerbach AD, Adler B, Chaganti RSK (1981) Prenatal and postnatal diagnosis and carrier detection of Fanconi's anaemia by a cytogenetic method. Pediatrics 67:128-135

13. Kwee ML, Poll EHA, van de Kamp JJP, de Koning H, Eriksson AW, Joenje H (1983) Unusual response to bifunctional alkylating agents in a case of Fanconi anemia. Hum Genet 64:384-387 
14. Gross M, Hanenberg H, Lobitz S, Friedl R, Herterich S, Dietrich R, Gruhn B, Schindler D, Hoehn H (2002) Reverse mosaicism in Fanconi anemia: natural gene therapy via molecular self-correction. Cytogenet Genome Res 98(2-3):126-135

15. Kalb R, Neveling K, Hoehn H, Schneider H, Linka Y, Batish SD et al (2007) Hypomorphic mutations in the gene encoding a key Fanconi anemia protein, FANCD2, sustain a significant group of FA-D2 patients with severe phenotype. Am J Hum Genet 80:895910

16. Waisfisz Q, Morgan NV, Savino M, deWinter J, van Berkel CG, Hoatlin ME et al (1999) Spontaneous functional correction of homozygous Fanconi anaemia alleles reveals novel mechanistic basis for reversion mosaicism. Nat Genet 22:379-383

17. Pasmooij AM, Jonkman MF, Uitto J (2012) Revertant mosaicism in heritable skin diseases: mechanism of natural gene therapy. Discov Med 14(75):167-179

18. Ellis NA, Ciocci S, German J (2001) Back mutation can produce phenotype reversion in bloom syndrome somatic cells. Hum Genet 108:167-173

19. Ariga T, Yamada M, Skiyama Y, Tatsuzawa O (1998) A case of Wiskott-Aldrich syndrome with dual mutations in exon 10 of the WASP gene: an additional de novo one base insertion, which restores frame shift due to an inherent one base deletion, detected in the major population of the patient's peripheral blood lymphocytes. Blood 92:699-701

20. Stephan V, Wahn V, Le Diest F, Dirksen U, Broker B, MullerFleckenstein I et al (1996) Atypical X-linked severe combined immunodeficiency due to possible spontaneous reversion of the genetic defect in T-cells. N Engl J Med 335:1563-1567

21. Hirschhorn R, Yang DR, Puck JM, Huie ML, Jiang CK, Kurlandsky LE (1996) Spontaneous in vivo reversion to normal of an inherited mutation in a patient with adenosine deaminase deficiency. Nat Genet 13:290-295

22. Kvittingen EA, Rootwelt H, Berger R, Brandtzaeg P (1994) Selfinduced correction of the genetic defect in tyrosinemia type I. J Clin Investig 94:1657-1661

23. Jonkman MF, Scheffer H, Stulp R, Pas HH, Nijenhuis M, Heeres K, Owaribe K, Pulkkinen L, Uitto J (1997) Revertant mosaicism in epidermolysis bullosa caused by mitotic gene conversion. Cell 88 : 543-551

24. Venugopal P, Moore S, Lawrence DM, George AJ, Hannan RD, Bray SCE, To LB, D'Andrea RJ, Feng J, Tirimacco A, Yeoman AL, Young CC, Fine M, Schreiber AW, Hahn CN, Barnett C, Saxon B, Scott HS (2017) Self-reverting mutations partially correct the blood phenotype in a Diamond Blackfan anemia patient. Haematologica 102:e506-e509

25. Jongmans MCJ, Verwiel ETP, Heijdra Y, Vulliamy T, Kamping EJ, Hehir-Kwa JY et al (2012) Revertant somatic mosaicism by mitotic recombination in dyskeratosis congenita. Am J Hum Genet 90: 426-433

26. Asur RS, Kimble DC, Lach FP, Jung M, Donovan FX, Kamat A, Noonan RJ, Thomas JW, Park M, Chines P, Vlachos A, Auerbach AD, Smogorzewska A, Chandrasekharappa SC (2018) Somatic mosaicism of an intragenic FANCB duplication in both fibroblast and peripheral blood cells observed in a Fanconi anemia patient leads to milder phenotype. Mol Genet Genomic Med 6:77-91

27. Youssoufian H (1996) Natural gene therapy and the Darwinian legacy. Nat Genet 13:255-256

28. Mankad A, Taniguchi T, Cox B, Akkari Y, Rathbun RK, Lucas L, Bagby G, Olson S, D'Andrea A, Grompe M (2006) Natural gene therapy in monozygotic twins with Fanconi anemia. Blood 107: 3084-3090

29. Poole SR, Smith AC, Hays T, McGavran L, Auerbach AD (1992) Monozygotic twin girls with congenital malformations resembling Fanconi anemia. Am J Med Genet 42:780-784
30. Kamimae-Lanning AN, Goloviznina NA, Kurre P (2013) Fetal origins of hematopoietic failure in a murine model of Fanconi anemia. Blood 121(11):2008-2012

31. Suzuki S, Racine RR, Manalo NA, Raffel GD (2017) Impairment of fetal hematopoietic stem cell function in the absence of Fancd2. Exp Hematol 48:79-86

32. Battaile KP, Bateman RL, Mortimer D, Mulcahy J, Rathbun RK, Bagby G, Fleming WH, Grompe M (1999) In vivo selection of wild-type hematopoietic stem cells in a murine model of Fanconi anemia. Blood 94:2151-2158

33. Tulpule A, Lensch MW, Miller JD, Austin K, D'Andrea A, Schlaeger TM, Shimamura A, Daley GQ (2010) Knockdown of Fanconi anemia genes in human embryonic stem cells reveals early developmental defects in the hematopoietic lineage. Blood 115(17):3453-3462

34. Raya A, Rodriguez-Piza I, Geunechea G, Vassena R, Navarro S, Barrero MJ et al (2009) Disease-corrected hematopoietic progenitors from Fanconi anaemia induced pluripotent stem cells. Nature 460(7251):53-59

35. Río P, Navarro S, Guenechea G, Sanchez-Dominguez R, Lamana ML, Yanez R et al (2017) Engraftment and in vivo proliferation advantage of gene-corrected mobilized CD34+ cells from Fanconi anemia patients. Blood 130(13):1535-1542

36. Rio P, Navarro S, Wang W, Sanchez-Dominguez PR, Segovia JC et al (2019) Successful engraftment of gene-corrected hematopoietic stem cells in non-conditioned patients with Fanconi anemia. Nat Med 25:1396-1401

37. Gregory JJ Jr, Wagner JE, Verlander PC, Levran O, Batish SD, Eide CR et al (2001) Somatic mosaicism in Fanconi anemia: evidence of genotypic reversion in lymphohematopoietic stem cells. Proc Natl Acad Sci U S A 98:2532-2537

38. Soulier J, Leblanc T, Largher J, Dastot H, Shimamura A, Guardiola $P$ et al (2005) Detection of somatic mosaicism and classification of Fanconi anemia patients by analysis of the FA/BRCA pathway. Blood 105:1329-1336

39. Trujillo Quintero JP (2013) Genética clínica la anemia de Fanconi. Dissertation, Universitat Autónoma de Barcelona. Doctoral thesis; Autonomous University of Barcelona. https://ddd.uab.cat/pub/tesis/ 2013/hdl 10803 129106/jptq1de1.pdf

40. Tolar J, Adair JE, Antoniou M, Bartholomae CC, Becker PS, Blazer BR (2011) Stem cell gene therapy for Fanconi anemia: report from the $1^{\text {st }}$ international Fanconi anemia gene therapy working group meeting. Mol Ther 19(7):1193-1198

41. Adair JE, Sevilla J, Heredia CD, Becker PS, Kiem HP, Bueren J (2017) Lessons learned from two decades of clinical trial experience in gene therapy for Fanconi anemia. Curr Gene Ther 16(5): 338-348

42. Rickman KA, Lach FP, Abhyankar A, Donovan FX, Sanborn EM, Kennedy JA, Sougnez C, Gabriel SB, Elemento O, Chandrasekharappa SC, Schindler D, Auerbach AD, Smogorzewska A (2015) Deficiency of UBE2T, the E2 ubiquitin ligase necessary for FANCD2 and FANCI ubiquitination, causes FA-T subtype of Fanconi anemia. Cell Rep 12(1):35-41

43. Virts EL, Jankowska A, Mackay C, Glaas MF, Wiek C, Kelich SL, Lottmann N, Kennedy FM, Marchal C, Lehnert E, Scharf RE, Dufour C, Lanciotti M, Farruggia P, Santoro A, Savasan S, Scheckenbach K, Schipper J, Wagenmann M, Lewis T, Leffak M, Farlow JL, Foroud TM, Honisch E, Niederacher D, Chakraborty SC, Vance GH, Pruss D, Timms KM, Lanchbury JS, Alpi AF, Hanenberg H (2015) AluY-mediated germline deletion, duplication and somatic stem cell reversion in UBE2T defines a new subtype of Fanconi anemia. Hum Mol Genet 24(18):5093-5108

44. MacMillan ML, Auerbach AD, Davies SD, DeFor TE, Gillio A, Giller R et al (2000) Hematopoietic cell transplantation in patients with Fanconi anemia using alternate donors: results of a total body irradiation dose escalation trial. Br J Haematol 109:121-129 
45. Bottega R, Nicchia E, Cappelli E, Ravera S, De Rocco D, Faleschini M et al (2018) Hypomorphic FANCA mutations correlate with mild mitochondrial and clinical phenotype in Fanconi anemia. Haematologica 103(3):417-426

46. Bagby $\mathrm{G}$ (2018) Recent advances in understanding hematopoiesis in Fanconi Anemia. F1000 Res 105. https://doi.org/10.12688/ f1000research.13213.1

47. Van Wassenhove LD, Mochly-Rosen D, Weinberg K (2016) Aldehyde dehydrogenase 2 in aplastic anemia, Fanconi anemia and hematopoietic stem cells. Mol Genet Metab 119(1-2):28-36

48. Hira A, Yabe H, Yoshida K, Okuno Y, Shiraishi Y, Chiba K, Tanaka H, Miyano S, Nakamura J, Kojima S, Ogawa S, Matsuo K, Takata M, Yabe M (2013) Variant ALDH2 is associated with accelerated progression of bone marrow failure in Japanese Fanconi anemia patients. Blood 122(18):3206-3209

49. Paustian L, Chao MM, Hanenberg H, Schindler D, Neitzel H, Kratz CP, Ebell W (2016) Androgen therapy in Fanconi anemia: a retrospective analysis of 30 years in Germany. Pediatr Hematol Oncol 33(1):5-12
50. Liu JM, Kim S, Read EJ, Futaki M, Dokal I, Carter CS, Leitman SF, Pensiero M, Young NS, Walsh CE (1999) Engraftment of hematopoietic progenitor cells transduced with the Fanconi anemia group C gene (FANCC). Hum Gene Ther 10(14):2337-2346

51. Kelly PF, Radtke S, von Kalle C, Balcik B, Bohn K, Mueller R, Schuesler T, Haren M, Reeves L, Cancelas JA, Leemhuis T, Harris R, Auerbach AD, Smith FO, Davies SM, Williams DA (2007) Stem cell collection and gene transfer in Fanconi anemia. Mol Ther 15(1):211-219

52. Zink F, Stacey SN, Norddahl GL, Frigge ML, Magnusson OT, Jonsdottir I et al (2017) Clonal hematopoiesis, with and without candidate driver mutations, is common in the elderly. Blood 130(6):742-752

53. Steensma DP (2018) Clinical consequences of clonal hematopoiesis of indeterminate potential. Blood Adv 2:264-269

Publisher's note Springer Nature remains neutral with regard to jurisdictional claims in published maps and institutional affiliations. 